\title{
Effects of Functional Group Interactions on the Bimolecular and Dissociation Reactions of Diols
}

\author{
Erika S. Eichmann, Erwin Alvarez, and Jennifer S. Brodbelt \\ Department of Chemistry, University of Texas, Austin, Texas, USA
}

\begin{abstract}
The influence of functional group interactions on the bimolecular and dissociation reactions of diols were examined in a quadrupole ion trap mass spectrometer. Reactions of dimethyl ether ions with diols resulted in formation of $(M+H)^{+}$ions and $(M+13)^{+}$ions (by net methyne addition). The product distribution depended on the relative separation of the hydroxyl groups within each diol, with the more proximate diols producing the greatest abundance of $(\mathrm{M}+13)^{+}$ions compared to $(\mathrm{M}+\mathrm{H})^{+}$ions. The enhancement of the formation of $(M+13)^{+}$ions is attributed to the capability for electrostatic interactions between the hydroxyl groups and the electropositive methylene group of the methoxymethylene reagent ion. The enhancement is most significant for diols that can adopt five- or to a lesser extent six-membered ring transition states (i.e., any 1,2 or 1,3 diol). Collision-activated dissociation (CAD) techniques, including both sequential activation experiments (MS ${ }^{n}$ ) and comparison of CAD spectra for model compounds, suggest that the $(\mathrm{M}+13)^{+}$ions are protonated cyclic diethers. (J Am Soc Mass Spectrom 1992, 3, 535-542)
\end{abstract}

$\mathrm{H}$ istorically, the influence of neighboring-group interactions has been an active area of research in organic chemistry in solution [1]. Likewise, there have been numerous accounts of the importance of functional group interactions of many types of difunctional molecules [2-18], including diols [2-12], diacids [13], diesters [14], and amino alcohols [15], in gas-phase reactions with positive ions. Perhaps the most relevant to the present work are those involving the interactions between hydroxyl groups. The hydroxyl group is one of the most ubiquitous and important functional groups found in biologically active compounds, such as sugars and steroids [19]. The hydroxyl group creates a nucleophilic site in molecules and may participate in hydrogen-bonding interactions. Simple diols can serve as first-order models of more complex biologically relevant molecules that contain multiple hydroxyl groups. With the advent of new volatilization methods that permit analysis of larger and more structurally complex molecules by mass spectrometry, it is of increasing interest to examine in detail the gas-phase bimolecular and dissociation reactions of diols to obtain a fundamental understanding of these important difunctional species and to develop analytical methods for characterizing related types of biomolecules.

Address reprint requests to Jennifer S. Brodbelt, Department of Chemistry, University of Texas, Austin, TX 78712-1167.
There have been numerous mass-spectrometric investigations of diols, mostly cyclic ones [2-12]. Some of these studies have concerned the influence of hydroxyl group interactions on the mass spectra generated by electron ionization (EI) or chemical ionization (CI) of various diols. For example, it was found that the EI-induced dehydration of stcroidal diols occurred only for 1,2 diols or 1,3 diols, not for 1,4 or even less proximal diols [2]. From the isobutane chemical ionization spectra of cyclic diols it was determined that the intensity of the peaks due to protonated molecules in the spectra was influenced by the stereochemical configuration of the diols and the relative proximity of the hydroxyl groups [3, 4], which promoted proton bridging. For instance, protonated cis-1,2-cyclopentanediol was found to be more stable (and less likely to dehydrate) than protonated trans-1,2-cyclopentanediol [3]. Analogous effects concerning the propensity for dehydration were noted in the collision-activated dissociation (CAD) mass spectra of (M $-\mathrm{H})^{-}$ions of diols $[10,11]$ formed by $\mathrm{HO}^{-}$chemical ionization. More recently, it was reported that stereoisomeric cyclic diols could be distinguished on the basis of endothermic or exothermic proton transfer reactions with ammonia in the collision quadrupole of a triple-quadrupole mass spectrometer [12]. The ammonium adduct ion was more abundant for trans1,2-cyclopentanediol than its cis-isomer because intramolecular solvent displacement resulted in elim- 
ination of $\mathrm{NH}_{3}$ from the cis-diol complex, due to the higher propensity for intramolecular hydrogen bonding.

We have undertaken a systematic study to evaluate the effects of various functional group interactions on ion-molecule reactions of gas-phase ions, and herein we report specifically on the bimolecular and dissociation reactions of simple diols. These reactions of the series of diols with dimethyl ether ions were examined in a quadrupole ion trap mass spectrometer. Product ions were characterized via CAD techniques and by comparison with the fragmentation behavior of model compounds. The primary objectives were to correlate diol functional group interactions with the product distributions of selected ion-molecule reactions involving dimethyl ether ions and to determine if the diols and their structurally modified product ions dissociated in ways that reflected differences in functional group interactions.

\section{Experimental}

Ion-molecule reactions were examined by using a Finnigan quadrupole ion trap mass spectrometer (ITMS) [20, 21]. A typical ion-molecule reaction sequence was initiated with a short electron ionization pulse, after which a selected reagent ion was isolated by using the appropriate application of $\mathrm{dc}$ and radiofrequency $(\mathrm{RF})$ voltages (apex isolation mode [22]). The dc voltage used to isolate a reagent ion was typically -40 to $-50 \mathrm{~V}$, and the RF voltage was about $500 \mathrm{~V}$ at $1.1 \mathrm{MHz}$. The chosen reagent ion was then allowed to undergo ion-molecule reactions (at $q=0.2$ ) with the neutral analyte for a period of $10-1000 \mathrm{~ms}$. The product ion spectrum was then recorded by using the mass-selective instability mode to eject ions from the trap into an electron multiplier. Alternatively, all reagent ions were allowed to react with neutral diols, and then a particular product ion was isolated prior to collisional activation. Collisional activation involved the application of an ac voltage of $0.3-1.0 \mathrm{~V}_{p-p}$ at the axial frequency of motion of the ion of interest (120 $\mathrm{kHz}$ at $q=0.3$ ). The dimethyl ether pressure was nominally $1 \times 10^{-5}$ torr, and the helium buffer gas pressure was $0.8 \mathrm{mtorr}$. Compounds were introduced via a leak valve or on a solids probe to $8 \times 10^{-7}$ torr. Typical ion-molecule reaction times were $50 \mathrm{~ms}$, and activation times were $4 \mathrm{~ms}$. All diols and model compounds ( $>95 \%$ purity) were obtained from Aldrich (Milwaukee, WI), and dimethyl ether was obtained from MG Industries.

\section{Results and Discussion}

\section{Ion-Molecule Reactions of Dimethyl Ether Ions with Diols}

The bimolecular reactions of ions generated from dimethyl ether with mono- and difunctional organic substrates have been studied previously [23], and two reactive ions are of particular interest. Protonated dimethyl ether, $\left(\mathrm{CH}_{3}\right)_{2} \mathrm{OH}^{+}$, at $\mathrm{m} / \mathrm{z} 47$, undergoes mildly exothermic proton transfer [24] with many organic substrates and does not promote extensive fragmentation of diols. ${ }^{*}$ The other reactive ion of interest from dimethyl ether, $\mathrm{CH}_{3} \mathrm{OCH}_{2}^{+}$at $m / z 45$, has been shown to induce methylene substitution reactions of organic substrates $(\mathrm{M})$ via elimination of methanol from the $\left(\mathrm{M}+\mathrm{CH}_{3} \mathrm{OCH}_{2}\right)^{+}$complex, or methyl cation transfer via loss of formaldehyde from $\left(\mathrm{M}+\mathrm{CH}_{3} \mathrm{OCH}_{2}\right)^{+}$, resulting in product ions at $(\mathrm{M}+$ $13)^{+}$or $(\mathrm{M}+15)^{+}$, respectively [23]. For the present study, the dimethyl ether ions were allowed to react with each diol during an ion-molecule reaction interval, resulting in products at $(\mathrm{M}+\mathrm{H})^{+}$and $(\mathrm{M}+13)^{+}$. Simple methyl cation transfer from the dimethyl ether ions to the diols does not occur and thus will not be discussed further.

A representative mechanism for reaction of methoxymethylene cation with 1,2-propanediol is suggested in Scheme I. The methylene substitution likely proceeds via initial formation of an ion-molecule complex of $\mathrm{CH}_{3} \mathrm{OCH}_{2}^{+}$and the diol (see Scheme I), with the primary electrostatic interactions occurring between the lone pairs of electrons on the oxygen atoms of the diol and the positively polarized methylene group of the methoxymethylene cation. The reaction proceeds by nucleophilic attack on the $\mathrm{CH}_{3} \mathrm{OCH}_{2}^{+}$by either oxygen atom of the diol, resulting in a complex, $\left(\mathrm{M}+\mathrm{CH}_{3} \mathrm{OCH}_{2}\right)^{+}$, that is not observed as a stable species in the quadrupole ion trap. ${ }^{\dagger}$ Elimination of methanol occurs spontaneously by abstraction of a proton from an oxygen atom by the methoxyl group, resulting in the product ion at $(M+13)^{+}$. Stabilization of the acyclic product ion may be provided by intramolecular interaction of the unmodified hydroxyl group with the positively polarized methylene end group, resulting in a cyclic structure. It is possible that the methoxyl group may abstract a proton from either oxygen site, but either case would allow intramolecular cyclization to occur.

For each diol with the hydroxyl groups located at symmetrical positions in the molecule (such as 1,2ethanediol, 1,3-propanediol, 2,3-butanediol, and 1,4butanediol), attack at either hydroxyl group would result in the same cyclic product. For 1,2-propanediol, 1,2-butanediol, and 1,3-butanediol, however, two different cyclic products are formed, based on attack on the methoxymethylene cation at either of the two nucleophilic oxygen sites. For each of these diols, the ring sizes of the resulting products are the same, but

\footnotetext{
* The proton affinity of dimethyl ether has been measured as 192.1 $\mathrm{kcal} / \mathrm{mol}$, and the proton affinities of the diols are estimated as 205-215 kcal/mol [24].

${ }^{\dagger}$ The $(M+45)^{+}$adduct has been formed in a conventional chemical ionization source of a triple-quadrupole mass spectrometer and gives rise to the $(\mathrm{M}+13)^{+}$product ion via loss of methanol after collisional activation.
} 


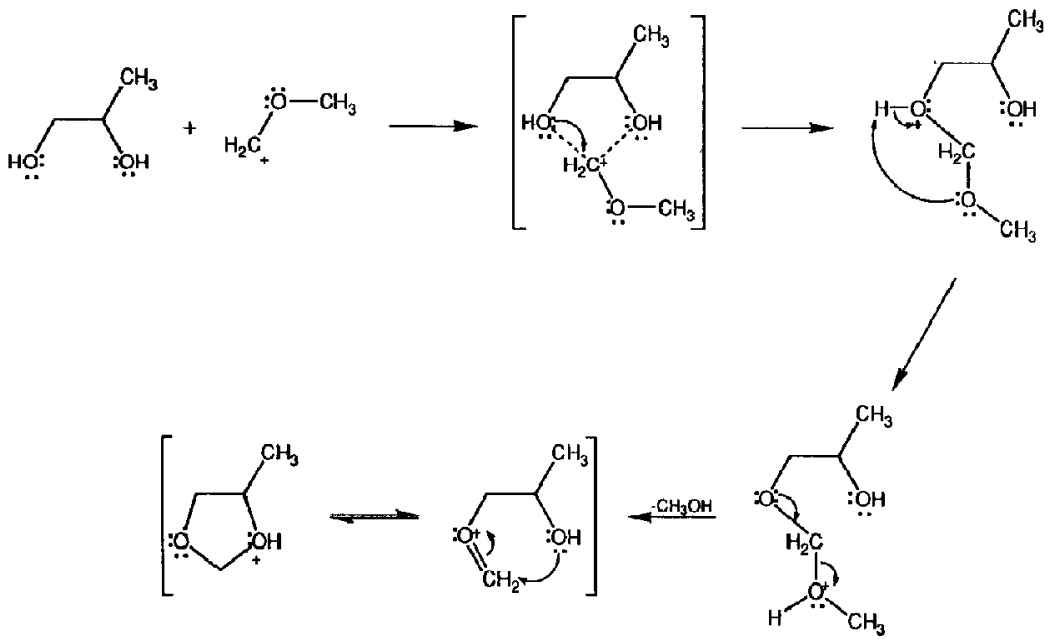

Scheme I. Proposed mechanism for formation of $(M+13)^{+}$ions of 1,2-propanediol.

the position of the alkyl substituent relative to the protonated ether site in the cyclic product changes. For simplicity, only the pathway representing attack by the terminal hydroxyl group is shown in Scheme I.

The relative product distributions of ions generated from ion-molecule reactions with dimethyl ether ions are listed in Table 1 for eight diols having variable hydroxyl group separation and carbon chain length. As the number of carbon atoms formally separating the hydroxyl groups increases, the extent of methylene substitution as compared to proton addition decreases. For example, methylene substitution and proton addition are equally favorable for 1,2ethanediol and 1,2-propanediol, but no methylene substitution at all is observed for 1,5-pentanediol or 1,6-hexanediol. When the total carbon chain length is held constant but the interfunctional distance is varied, as represented by the butanediol series, the formation of $(\mathrm{M}+13)^{+}$decreases in the order 1,2 butanediol $\simeq 2,3$-butanediol $>1,3$-butanediol $>1,4$ butanediol. These results indicate that the proximity of

Table 1. Product distributions for ion-molecule reactions of diols with dimethyl ether ions

\begin{tabular}{lcc}
\hline \multicolumn{1}{c}{ Diol } & \multicolumn{2}{c}{$\%$ Total ion current } \\
\cline { 2 - 3 } & $(\mathrm{M}+\mathrm{H})^{+}$ & $(\mathrm{M}+13)^{+}$ \\
\hline \hline 1,2-Ethanediol & 50 & 50 \\
1,2-Propanediol & 50 & 50 \\
1,3-Propanediol & 70 & 30 \\
1,2-Butanediol & 60 & 40 \\
2,3-Butanediol & 60 & 40 \\
1,3-Butanediol & 70 & 30 \\
1,4-Butanediol & 85 & 15 \\
1,5-Pentanediol & 100 & 0 \\
1,6-Hexanediol & 100 & 0 \\
\hline
\end{tabular}

the functional groups has a dramatic effect on the favorability of competing reactions.

The ion-molecule product distributions for reactions of monofunctional alcohols with dimethyl ether ions were also examined. Like the diols with large interfunctional distances, simple alcohols (1-propanol, 1-butanol, ethanol) exhibit no formation of $(\mathrm{M}+13)^{+}$ ions. This highlights the necessity for the presence of two proximate nucleophilic hydroxyl groups in a molecule in order for the methylene substitution reaction to be possible.

This observation can be rationalized in several ways. First, the favorability of the methylene substitution reactions for the diols with hydroxyl groups on adjacent carbon atoms may be influenced by secondary functional group participation by the proximate hydroxyl groups, as shown in Scheme I for 1,2-propanediol. Such interactions would also stabilize the ion-molecule complex initially formed during the bimolecular reaction between $\mathrm{CH}_{3} \mathrm{OCH}_{2}^{+}$and the diol (as shown in Scheme I) and then assist in stabilizing the resulting product ion by intramolecular cyclization. In fact, as evidenced from CAD spectra discussed later, the final product ions are likely cyclic in nature, and the stability of the product may be correlated with the tendency of the ion to cyclize. In terms of the effects of the interfunctional distance on the favorability of the methylene substitution reaction, diols that can adopt five-membered (especially favorable) or six-membered (somewhat less favorable) rings (i.e., any 1,2 or 1,3 diol) produce the greatest relative abundance of the $(\mathrm{M}+13)^{+}$ion, as shown in Table 1.

Another factor that must be considered is that simple proton transfer from protonated dimethyl ether to the diol may be thermochemically or kinetically favored over the reaction of the diol with $\mathrm{CH}_{3} \mathrm{OCH}_{2}^{+}$ 
for diols with higher gas basicities. The basicities of the diols are estimated to fall in the order observed for diamines [24]: 1,6-hexanediol $>1,5$-pentanediol $>$ 1,4-butanediol $>1,3$-butanediol $>$ 1,3-propanediol $>$ 1,2 ethanediol. In many cases, the trends in nucleophilicities of molecules parallel the orders of basicities, and thus one might expect the formation of both $(\mathrm{M}+\mathrm{H})^{+}$and $(\mathrm{M}+13)^{+}$to be increasingly favored for the larger diols. However, other factors must be considered when evaluating general reactivity trends, including the stabilities of the diol products, the kinetics of simple proton transfer involving protonated dimethyl ether versus the kinetics of methylene substitution involving $\mathrm{CH}_{3} \mathrm{OCH}_{2}^{+}$, and the tendency of the larger diols to undergo intramolecular cyclization via covalent bond formation, as in $(M+13)^{+}$, versus hydrogen bond formation, as in $(\mathrm{M}+\mathrm{H})^{+}$. In any case, the data in Table 1 suggest that the trends of basicity and nucleophilicity for the diols are not parallel.

\section{CAD of Protonated Diols}

Each protonated diol was collisionally activated to induce dissociation into structurally diagnostic fragment ions. The resulting spectra are summarized in Table 2. Only two dissociation pathways are observed: elimination of one or two molecules of water. For protonated 1,2-ethanediol, 1,2-propanediol, 1,3-propanediol, and 2,3-butanediol, the double dehydration fragment ion is absent or appears in relatively small amounts, whereas for protonated 1,3butanediol, 1,2-butanediol, 1,4-butanediol, 1,5pentanediol, and 1,6-hexanediol, the amount of the double dehydration product ion observed exceeds that of the single dehydration fragment.

The relative extents of single and double dehydration can be rationalized by consideration of the nature of the carbocations formed after the first stage of dehydration and of the favorability of the proton transfer required for the second dehydration step. In addition, it has been shown previously that the pinacol rearrangement may occur after dehydration of

Table 2. Relative extent of dehydration of collisionally activated protonated diols

\begin{tabular}{lcc}
\hline & \multicolumn{2}{c}{$\%$ Total ion current } \\
\cline { 2 - 3 } Diol & $-\mathrm{H}_{2} \mathrm{O}$ & $-2 \mathrm{H}_{2} \mathrm{O}$ \\
\hline 1,2-Ethanediol & 98 & 2 \\
1,2-Propanedial & 98 & 2 \\
1,3-Propanediol & 80 & 20 \\
1,2-Butanediol & 25 & 75 \\
2,3-Butanediol & 80 & 20 \\
1,3-Butanediol & 10 & 90 \\
1,4-Butanediol & 50 & 50 \\
1,5-Pentanediol & 40 & 60 \\
1,6-Hexanediol & 30 & 70 \\
\hline
\end{tabular}

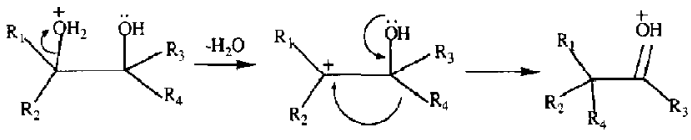

Scheme II. Mechanism of the pinacol rearrangement. $\left(R_{n}=\right.$ alkyl group or hydrogen.)

protonated diols that have the hydroxyl groups located on adjacent carbon atoms $[25,26]$. In the pinacol rearrangement, the carbocation formed after dehydration of the protonated diol is stabilized by 1,2-migration of an alkyl group or a hydrogen atom from the carbon atom possessing the remaining hydroxyl group. forming a protonated carbonyl product ion (illustrated in Scheme II).

For example, consider protonated 1,2-propanediol and 1,3-propanediol. Dehydration of protonated 1,3propanediol (which may initially be intramolecularly hydrogen-bonded) likely results in initial formation of a relatively unfavorable primary carbocation, as shown in Scheme III. For the second elimination of water, the hydroxyl group would presumably abstract a proton from the carbon atom adjacent to the carbocation, and the subsequent dehydration would result in an energetically favorable allylic cation. The process would occur through a four-centered transition state. In contrast, dehydration of protonated 1,2-propanediol may be followed by a pinacol rearrangement as shown in Scheme IIIb. Protonation of 1,2-propandiol likely results in an intramolecularly hydrogen-bonded ion, from which dehydration may occur involving either hydroxyl site. Migration of a hydrogen or methyl group from the adjacent carbon position to the carbocation site results in a stabilized protonated carbonyl product, shown in Scheme IIIb as protonated propionaldehyde. Subsequent dehydration is not favored. The fact that the $\mathrm{CAD}$ spectrum of protonated 1,3-propanediol shows greater double dehydration than the CAD spectrum of protonated 1,2-propanediol supports the plausibility of an operative pinacol rearrangement. Further support for the pinacol rearrangement was obtained by performing MS/MS/MS (sequential collisional activation) experiments of selected precursor ions [21] and comparison of CAD spectra of model compounds. MS/MS/MS experiments consist of activation of a selected precursor ion to induce dissociation, then activation of a resulting fragment ion in a second stage of collisional activation. For example, protonated 1,2-propanediol was isolated and collisionally activated to induce dehydration to $\mathrm{m} / \mathrm{z} 59$ (MS/MS). Then the fragment of $\mathrm{m} / z \quad 59$ was isolated and collisionally activated (MS/MS/MS), and it dissociated to fragments at $\mathrm{m} / \mathrm{z}$ 31, 39, and 41. This MS/MS/MS spectrum matches that obtained from $C A D$ of protonated propionaldehyde but does not match the CAD spectra of many other isomeric $\mathrm{C}_{3} \mathrm{H}_{9} \mathrm{O}^{+}$ions [27].

The rationalization of the relative extent of double dehydration for the other protonated diols follows 
(a)

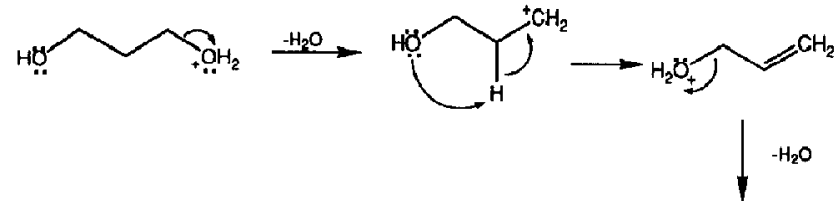

(b)

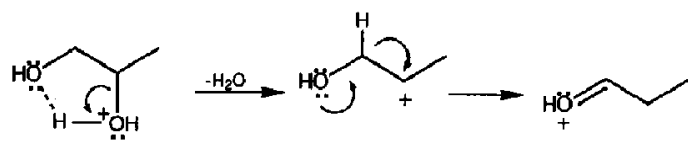

Scheme III. (a) Mechanism for dissociation of protonated 1,3-propanediol. (b) Mechanism for dissociation of protonated 1,2-propanediol. similar logic. For example, dehydration of protonated 1,2-ethanediol would result in a primary carbocation that could easily tautomerize to the keto form via the pinacol rearrangement (see Scheme IVa), and therefore little double dehydration is expected. In addition, loss of a second water molecule would result in the formation of the highly unstable $\mathrm{CH}_{2}=\mathrm{CH}^{+}$ion. Indeed, no appreciable amount of this fragment is formed (see Table 1). A similar argument explains the low extent of double dehydration for protonated 2,3butanediol relative to 1,3- and 1,4-butanediol. For the 2,3-isomer, the furst dehydration step results in a carbocation that can be sufficiently stabilized by migration of a methyl group via a pinacol rearrangement and formation of protonated isobutyraldehyde (Scheme IVb). MS/MS/MS/MS experiments support this mechanism. First, protonated 2,3-butanediol $(\mathrm{m} / \mathrm{z}$ 91) was isolated and activated (MS/MS) to induce dehydration to $m / z$ 73. Then the fragment at $m / z 73$ was isolated and activated (MS/MS/MS), causing dissociation to only $m / z 55$ via dehydration. Next the fragment at $m / z \quad 55$ was isolated and activated (MS/MS/MS/MS), producing fragment ions at $\mathrm{m} / \mathrm{z}$ $29(50 \%)$ by elimination of $\mathrm{C}_{2} \mathrm{H}_{2}, m / z 39(25 \%)$ by loss of methane, $m / z 53(15 \%)$, and $m / z 27(10 \%)$. This spectrum matches the CAD spectrum obtained for protonated isobutyraldehyde. Alternately, double dehydration is the predominant dissociation process for protonated 1,3-butanediol. The first dehydration results in a relatively stable secondary carbocation. The second dehydration reaction would then be initiated by proton transfer through a highly favorable six-membered cyclic transition state and is thus particularly favored for this compound (see Scheme IVc).

Isomerization of the various dehydration product ions via nonspecific hydrogen and methyl migrations to form more stable secondary or tertiary alkyl ions is a possibility that cannot be ruled out by examination of the spectral data. Typically, collisional activation results in fragment ions favored under kinetic control [15], and isomerization to the thermodynamic product is not likely to be significant. In addition, the fact that the relative extents of dehydration and double dehydration for each diol are different indicates that each isomeric diol retains distinctive dissociation characteristics and suggests that if isomerization is occurring, it is not complete.

\section{CAD of $(M+13)^{+}$of Diols}

For each diol that reacted with dimethyl ether ions to form products at $(\mathrm{M}+13)^{+}$due to $\left(\mathrm{M}+\mathrm{CH}_{3} \mathrm{OCH}_{2}\right.$ $\left.-\mathrm{CH}_{3} \mathrm{OH}\right)^{+}, \mathrm{CAD}$ spectra were acquired to assist in

(a)

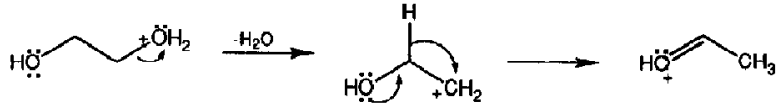

(b)<smiles>CC(=O)C(C)[OH2+]</smiles><smiles></smiles><smiles>C[C+]1CCCC1(C)O</smiles><smiles>CC(C)C=[OH+]</smiles>

(c)<smiles>CC(C)CCO</smiles><smiles>C1CCCCCC1</smiles><smiles>CCCCCCC</smiles>

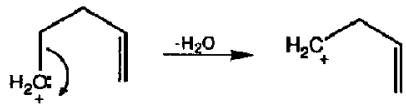

Scheme IV. (a) Mechanism for dehydration of protonated 1,2-ethanediol. (b) Mechanism for dehydration of protonated 2,3-butanediol. (c) Mechanism for double dehydration of protonated 1,3-butanediol. 
the structural characterization of the $(\mathrm{M}+13)^{+}$product ions. The major fragments of these product ions are listed in Table 3. Unlike the simple CAD spectra observed for the protonated diols, the CAD spectra of the $(M+13)^{+}$ions indicate the existence of many competitive fragmentation routes. Several striking features are apparent. First, for three of the diols with adjacent hydroxyl groups (1,2-propanediol, 2,3butanediol, and 1,2-butanediol), the two predominant dissociation routes are loss of formaldehyde and loss of formaldehyde + water. For the diols with greater functional group separation (1,3-propanediol, 1,4butanediol, 1,3-butanediol), the three most most significant fragmentation pathways are elimination of methanol, elimination of water, and loss of $44 \mathrm{u}$.

To further characterize the structures of the $(\mathrm{M}+$ $13)^{+}$ions, the CAD spectra of model ions were compared to the CAD spectra of the diol ions, and $\mathrm{MS}^{n}$ (sequential activation) experiments were done for more detailed characterization of dissociation pathways. First, ions were generated from 3-ethoxy-1propanol (EI-induced alpha cleavage of the terminal methyl radical) and from protonation of 1,3-dioxane to model the $(M+13)^{+}$ion of 1,3-propanediol (see Scheme V). The CAD spectra of these ions are shown in Figure 1, along with the CAD spectrum of the 1,3-propanediol $(M+13)^{+}$ion. Whereas the spectrum for the acyclic model is significantly different, the spectra for the 1,3-dioxane model and the 1,3propanediol are satisfactory matches, suggesting that the cyclic form of the $(M+13)^{+}$ion does indeed predominate over the acyclic form. Furthermore, the MS/MS/MS experiments performed for each fragment ion seen in Figure $1 \mathrm{a}$ and $\mathrm{b}$ give identical spectra. For example, sequential isolation and activation of the ion of $m / z 71$ produces fragment ions at $m / z 41$, 43 , and 39. The sequential activation of the ion of $\mathrm{m} / \mathrm{z}$
59 produces predominantly the ion of $m / z 31$ with some $m / z 41$. Finally, the fragment ion of $m / z 57$ dissociates to ions at $m / z 27$ and 29. Likewise, the CAD spectrum recorded for protonated 1,3-dioxolane, a five-membered ring diether, reproduces the one obtained for $(M+13)^{+}$of 1,2-ethanediol. In each case, loss of formaldehyde is the sole dissociation route.

For the butanediols, one cyclic model compound was available. The CAD spectrum for protonated 4 methyl-1,3-dioxane shows that it dissociates via the same routes (and with similar fragment distributions) as the $(\mathrm{M}+13)^{+}$ion of 1,3-butanediol. Moreover, the CAD spectra obtained by MS/MS/MS experiments of each of the fragment ions (i.e., $m / z 71$ and 59) duplicate the spectra obtained for the corresponding diol fragment ions. These results support the type of cyclic product structure proposed in Scheme I.

The structures of the fragment ions produced from collisional activation of the various $(M+13)^{+}$ions are rationalized by appropriate mechanisms. For example, the elimination of formaldehyde alone or in conjunction with dehydration occurs for most of the $(M$ $+13)^{+}$ions, particularly the more proximate diols $(1,2$ or 2,3$)$. As exemplified by 1,2-propanediol, the cyclic $(\mathrm{M}+13)^{+}$ion may undergo direct cleavage of formaldehyde, producing a secondary carbocation (see Scheme VI). Analogous mechanisms for the loss of formaldehyde can be envisioned for the dissociation of the $(\mathrm{M}+13)^{+}$ions of the other diols.

The structure of the ion resulting from direct cleavage of formaldehyde from the $(\mathrm{M}+13)^{+}$ion (as shown in Scheme VI) is identical to the primary dehydration product of corresponding protonated diol (indicated in Scheme IIIb). To support this assertion, the CAD spectra of the fragment ions formed by either dehydration of the protonated diol or loss of $30 \mathrm{u}$ from the $(M+13)^{+}$ion were compared.

Table 3. CAD spectra of $(M+13)^{+}$adducts of diols

\begin{tabular}{|c|c|c|c|c|c|c|}
\hline \multirow{2}{*}{$\begin{array}{c}\text { Diol } \\
(m / z \text { of } \\
\left.(M+13)^{+}\right)\end{array}$} & \multirow[b]{2}{*}{$-\mathrm{H}_{2} \mathrm{O}$} & \multirow[b]{2}{*}{$-\mathrm{CH}_{2} \mathrm{O}$} & \multicolumn{4}{|c|}{$\%$ Total ion current } \\
\hline & & & $-\left(\mathrm{CH}_{2} \mathrm{O}+\mathrm{H}_{2} \mathrm{O}\right\}$ & $-\mathrm{CH}_{3} \mathrm{OH}$ & -44 & -60 \\
\hline 1,2-Ethanediol & 0 & 100 & 0 & 0 & 0 & 0 \\
\hline \multicolumn{7}{|l|}{ (75) } \\
\hline 1,2-Prapanediol & 0 & 85 & 15 & 0 & 0 & 0 \\
\hline \multicolumn{7}{|l|}{ (89) } \\
\hline 1,3-Propanediol & 15 & 30 & 0 & 30 & 25 & 0 \\
\hline \multicolumn{7}{|l|}{ (89) } \\
\hline $\begin{array}{l}\text { 1,2-Butanediol } \\
(103)\end{array}$ & $<2$ & 5 & 75 & 5 & 10 & 0 \\
\hline 2,3-Butanediol & 0 & 85 & 15 & 0 & 0 & 0 \\
\hline \multicolumn{7}{|l|}{ (103) } \\
\hline 1,3-Butanediol & 0 & o & 0 & 60 & 20 & 20 \\
\hline \multicolumn{7}{|l|}{ (103) } \\
\hline 1,4-Butanediol & 30 & 30 & 10 & 0 & 30 & 0 \\
\hline$(103)$ & & & & & & \\
\hline
\end{tabular}


(a)

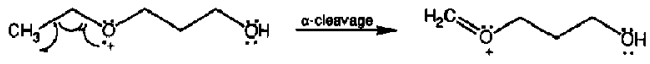

(b)

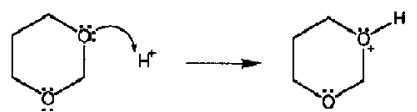

Scheme V. Structures of model ions generated from (a) 3 ethoxy-1-propanol and (b) 1,3-dioxane.

For example, the $m / z 59$ ions from the $(\mathrm{M}+\mathrm{H})^{+}$ and the $(\mathrm{M}+13)^{+}$ions of 1,2-propanediol were examined by MS/MS/MS experiments. First, protonated 1,2-propanediol was collisionally activated to produce its dehydration fragment ion at $m / z 59$.

(a)

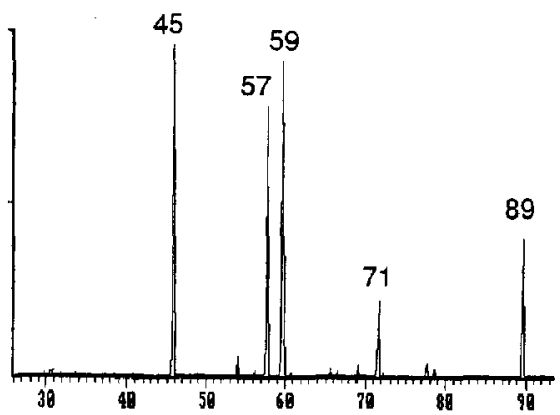

(b)

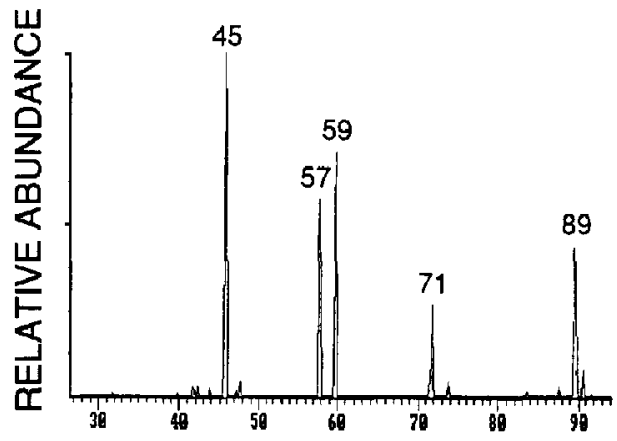

(c)

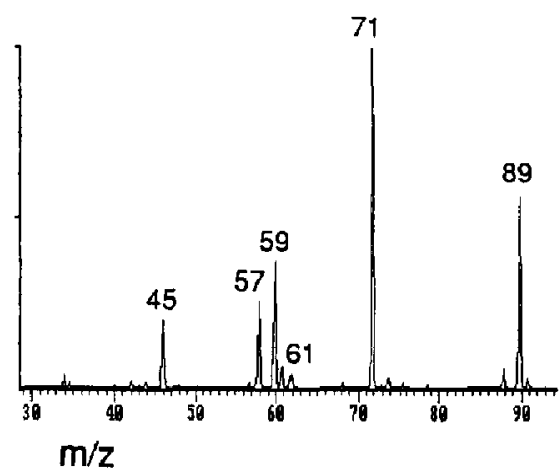

Figure 1, CAD spectrum of the $m / z 89$ ions from (a) the $(M+13)^{+}$ion of 1,3-propanediol, (b) protonated 1,3-dioxane, and (c) 3-ethoxy-1-propanol (alpha cleavage product).
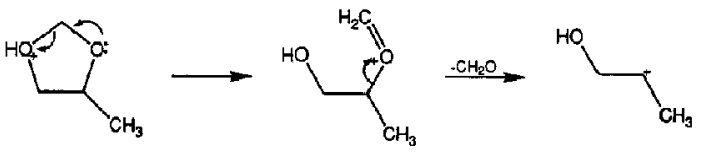

$89^{*}$

$59^{+}$

Scheme VI. Mechanism for dissociation of the $(M+13)^{+}$ion of 1,2-propanediol.

Then $m / z 59$ was isolated and activated (MS/MS/ MS). Next, the $(M+13)^{+}$ion was collisionally activated to promote dissociation to $m / z 59$, and this fragment ion was activated (MS/MS/MS). In both cases, fragment ions were observed at $m / z 31(45 \%)$, $m / z 41(45 \%)$, and $m / z 39(10 \%)$. The similar fragmentation patterns support the suggestion that dehydration of the protonated diol or loss of formaldehyde from the corresponding $(\mathrm{M}+13)^{+}$ion results in formation of similar fragment ion structures. The activation of $\mathrm{m} / \mathrm{z} 59$ results in extensive dehydration (formation of $m / z 41$ ), whereas the selective activation of protonated 1,2-propanediol does not result in extensive double dehydration (also formation of $\mathrm{m} / \mathrm{z}$ 41). This is not contradictory behavior. Rather, it indicates that the double dehydration of protonated 1,2propanediol is not energetically favored but can be induced by isolating the dehydration fragment ion and subjecting it to further stages of activation. In an ion trap, once an ion dissociates, the newly formed fragment ion is off-resonance for further activation and therefure will not be further excited. However, in MS/MS/MS experiments, a fragment ion may be specifically excited by application of on-resonance activation, and the dissociation of such a fragment ion does not necessarily reflect the favored dissociation pathways of the original precursor ion.

The trends for the relative extent of dehydration subsequent to loss of $\mathrm{CH}_{2} \mathrm{O}$ from the $(\mathrm{M}+13)^{+}$ions agree with those rationalized for the sequential dehydration of the protonated diols. For instance, the $(\mathrm{M}+13)^{+}$ion of 1,2-ethanediol shows no sequential loss of formaldehyde and water. Likewise, protonated 1,2-ethanediol undergoes minimal double dehydration. Similarly, the CAD spectrum of protonated 1,2butanediol indicates that $75 \%$ of the fragment ion current is due to double dehydration; the $\mathrm{CAD}$ spectrum of the $(M+13)^{+}$ion shows that the peak due to loss of formaldehyde and water accounts for $75 \%$ of the ion current. The 2,3-butanediol ions follow similar trends, but the $(M+13)^{+}$ion from 1,2-propanediol shows significantly more dehydration after $\mathrm{CH}_{2} \mathrm{O}$ loss than predicted.

For the diols with greater functional group separation, the losses of formaldehyde alone or in conjunction with water are less important fragmentation routes for the $(M+13)^{+}$ions. Instead, predominant pathways are elimination of methanol, water, or $44 \mathrm{u}$. This is attributed to the fact that these less proximate 
diols form $(\mathrm{M}+13)^{+}$adducts that have larger ring sizes (six or seven members) or perhaps do not cyclize compared to the 1,2 diols (five-membered rings). These factors presumably facilitate alternate dissociation routes.

\section{Conclusions}

Functional group interactions have proved to be particularly important in mediating the bimolecular and dissociation reactions of diols. The proximity of the functional groups is related to the relative favorability of the methylene substitution reactions and protonation reactions. The latter are favored for diols with large functional group separations. The fact that simple alcohols do not undergo methylene substitution supports the idea that two proximate nucleophilic hydroxyl groups are needed to promote the reaction. The methylene substitution reactions generate cyclic diol adduct ions that dissociate via routes that reflect the initial proximity of the hydroxyl groups.

\section{Acknowledgments}

Support from the Welch Foundation (F-1155), NSF (CHE9122699) an ACS.PRF grant (22270-G5), and an ASMS Young Investigator award is gratefully acknowledged.

\section{References}

1. Capon, B.; McManus, S. P. Neighboring Group Participation; Plenum Press; New York, 1976, vol 1.

2. Fenselau, C. C.; Robinson, C. H. I. Am. Chem. Soc. 1971, 93, 3070.

3. Reich, M.; Schwarz, H. Org. Mass Spectrom. 1977, 12, 566.

4. Clayes, M.; Van Haver, D. Org. Mass Spectrom. 1977, 12, 531 .

5. Respondek, J.; Schwarz, H.; Van Gaever, F.; Van de Sande, C. C. Org. Mass Spectrom. 1978, 13, 618.

6. Dhaenens, L.; Van de Sande, C. C; Van Gaever, F. Org. Mass Spectrom. 1979, 14, 145.
7. Winkler, F. J.; Stahl, D. J. Am. Chem. Soc. 1979, 101, 3685.

8. Blanc, P. A.; Gulacar, F. O.; Buchs, A. Org. Mass Spectrom. 1978, 13, 135.

9. Beloeil, J. C.; Bertranne, M.; Stahl, D.; Tabet, J. C. I. Am. Chem. Soc. 1983, 105, 1355.

10. Brumley, W. C.; Andrzejewski, D, Sphon, J. A. Org. Mass Spectrom. 1988, 23, 204.

11. Gaumann, T.; Stahl, D.; Tabet, J. C. Org. Mass Spectrom. $1983,18,263$.

12. Meyerhoffer, W. J.; Bursey, M. M. Org. Mass Spectrom. 1989, 24, 169.

13. Harrison, A. G.; Krisha Mohan Rao Kallury, R. Org. Mass Spectrom. 1980, 15, 277.

14. Fales, H. M.; Milne, G. W. A.; Nicholson, R. S. Anal. Chem. 1971, 43, 1785.

15. Wysocki, V. H.; Burinsky, D. J.; Cooks, R. G. J. Urg. Chem. $1985,50,1287$.

16. Morton, T. H.; Beauchamp, J. L. J. Am. Chem. Soc. 1972, 94, 3671 .

17. Aue, D. H.; Webb, H. M.; Bowers, M. T. I. Am. Chem. Soc. 1973, 95, 2699.

18. Dzidic, I.; McCloskey, J. A. I. Am. Chem. Soc. 1971, 93, 4955.

19. Rawn, J. D. Biochemistry; Harper and Row; New York, 1983.

20. Louris, J. N.; Cooks, R. G.; Syka, J. E. P.; Kelley. P. E.; Stafford, G. C.; Todd, J. F. J. Anal. Chem. 1987, 59, 1677.

21. Louris, J. N.; Brodbelt, J. S.; Cooks, R. G.; Glish, G. L.; Van Berkel, G. J.; Mcluckey, S. A. Int. J. Mass Spectrom. Ion Proc. 1990, 96, 117.

22. Weber-Grabau, M.; Kelley, P. E.; Syka, J. E. P.; Bradshaw, S. C.; Brodbelt, J. S. Proceedings of the 35th Annual Conference of the American Society for Mass Spectrometry, 1987, 1114.

23. Brodbelt, J. S.; Liou, C. C.; Donovan, T. L. Anal, Chem. 1991, 63, 1205.

24. Lias, S. G.; Bartmess, J. E.; Licbman, J. F.; Holmes, J. L.; Levin, R. D.; Mallard, W. G. J. Phys. Chem. Ref. Data 1988, 17, suppl 1.

25. Glish, G. L.; Cooks, R. G. I. Am. Chem. Soc, 1978, 100 , 6720 .

26. Maquestiau, A.; Flammang, R.; Flammang-Barbieux, M.; Mispreuve, H. Tetrahedron 1980, 36, 1993.

27. Brodbelt, J. S.; Cooks, R. G. Int. J. Mass Spectrom. Ion Proc. $1988,86,253$. 